\title{
A Multi-scale Numerical Study of the Flow, Heat, and Mass Transfer in Protective Clothing
}

\author{
Michal P. Sobera ${ }^{1,2}$, Chris R. Kleijn ${ }^{1}$, Paul Brasser ${ }^{2}$, and \\ Harry E.A. Van den Akker ${ }^{1}$ \\ 1 Kramers Laboratorium voor Fysische Technologie, Delft University of Technology, \\ Prins Bernhardlaan 6, 2628BW, Delft, The Netherlands \\ 2 TNO Prins Maurits Laboratory, P.O. Box 45, 2280AA, Rijswijk, The Netherlands
}

\begin{abstract}
A multi-scale study of the performance of protective clothing has been performed by coupling various types of numerical simulation of flow, heat and mass transfer. From meso-scale Direct Numerical Simulation, it was found that the flow underneath the clothing is laminar and periodic, with a magnitude much smaller than the free stream velocity. Micro-scale Direct Numerical Simulation revealed a simple relation between textile porosity and permeability. A good agreement was found between flow and heat transfer predictions of Direct Numerical Simulation and Reynolds Averaged simulation. From the latter, an engineering correlation for heat and mass transfer was deduced.
\end{abstract}

\section{Introduction}

The demands on NBC (Nuclear-Biological-Chemical) protective clothing are strict and mostly contradictory, requiring the combination of optimal protection and optimal comfort. This makes the design and development of effective NBC protective clothing complex. It is believed, that computational models of flow, heat and mass transfer can simplify this process. Flow, heat and mass transfer in NBC protective clothing depend on phenomena at very different scales, ranging from the sub-millimeter scales of textile fibers and turbulent flow structures, via the centimeter to decimeter scale of the flow around body parts, to the meter scale of a full clothed person. Therefore, a coupled multi-scale modeling approach is necessary. In this paper we describe the development of such an approach. Using Computational Fluid Dynamics (CFD), we study NBC protective clothing on different scales, focusing on different aspects of flow, heat and mass transfer. Information from small scales is used to extract lumped models for larger scales. Large-scale simulations are used to determine the conditions at which small scale simulations are performed.

\section{Multi Scale Modeling Strategy}

We aim to develop predictive models for the performance of protective clothing at full body scale. At this (macro-) scale it is not feasible to account for phenomena at the scale of the textile structure, nor is it possible to account for 
the detailed properties of the turbulent flow. Therefore, models are based on large-scale, time-averaged Computational Fluid Dynamics descriptions of the flow, using the so called Reynolds Averaged Navier Stokes (RANS) approach. The textile material is modeled as a porous material with particular macroscopic properties. This approach was used in our previous study [1, were we investigated the influence of flow and clothing properties on heat and mass transfer. Two aspects of this kind of simulation require more detailed studies at smaller scales: Firstly, the performance of RANS models in laminarizing flow through a porous material is unknown. Therefore, in this paper we extend and compare our previous RANS study with DNS at meso-scale. We perform a detailed study of the flow around a single clothed limb modeled as a circular cylinder sheathed by a porous layer (see Fig. 1) using DNS. From these simulations we evaluate the performance of RANS turbulence models for the flows of interest. Secondly, engineering models for the lumped porous properties of the clothing material can be deduced from micro-scale DNS analyzes of the flow through the actual geometry of the textile structure. Input from both the meso-scale DNS and the micro-scale textile studies are used to improve and validate RANS simulations.

\subsection{Meso-scale DNS}

The flow around a cylinder at subcritical Re has been studied by several authors [2345.67], both numerically and experimentally. In the range of Reynolds number $\mathrm{Re}=10^{3}$ to $10^{7}$ (based on cylinder diameter and free stream velocity), the flow is periodic and transitional in character. In the present work, the focus was on a subcritical flow regime $\left(10^{3} \leq \mathrm{Re} \leq 10^{5}\right.$, corresponding to air velocities of $0.1-10 \mathrm{~m} / \mathrm{s}$ around a typically sized limb) [2], in which, due to the vortex shedding the flow is strongly unsteady. The boundary layer remains fully laminar up to the separation point and transition to turbulence occurs in the wake.

The flow around a cylinder sheathed by a second, larger, porous cylinder (see Fig. 1), has received little attention. The general characteristics of the flow around a such an obstacle are expected to be similar to those of the flow around a single solid cylinder. However, not much is known about the interaction between the unsteady, turbulent flow outside the porous cylinder and flow inside the gap between the porous and the solid cylinder. For our DNS we used the commercial CFD solver Fluent 6, which is based on an unstructured finite volume formulation. Unstructured solvers have relatively high memory requirements (in our case $\approx 1$ GB of RAM per $1 \mathrm{M}$ of computational cells). In order to reduce the number of cells, local grid refinement was applied, with a fine mesh in the vicinity of the cylinder and in the near wake region. The grid spacing in this region was approximately $0.008 D$ similar to that reported by Tremblay [7, who studied the flow around a cylinder by means of DNS with a structured CFD code. In the far field we used a slightly lower grid resolution. As a result, the total number of cells in our simulations was $\sim 6 \cdot 10^{6}$, as compared to $\sim 40 \cdot 10^{6}$ in the simulations of Tremblay.

The porous material was treated as a fluid zone, where the pressure drop was imposed as a sink in the momentum equation according to Darcy's law. For its 

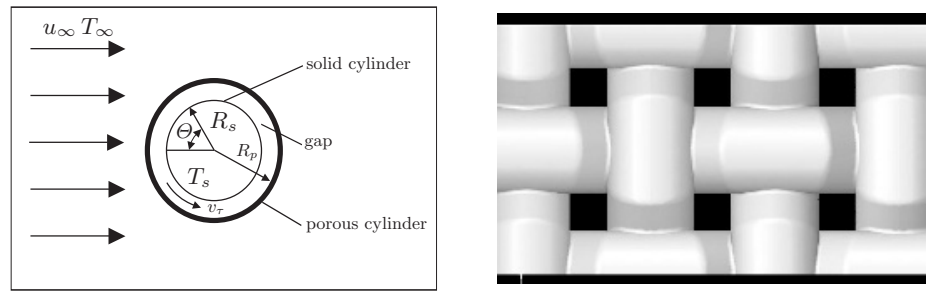

Fig. 1. Schematic problem description for meso-scale approach (left) and computational representation of the net of fibers (right)

resistance, values were set that were obtained experimentally from a sample of real protective clothing. The cylinder length was $\pi D$, with periodic boundaries at the end walls. Thus, in fact we simulated an infinitely long cylinder. The simulation was run with a constant time step $\Delta t=0.001 \frac{D}{u_{\infty}}$ and was carried out for 9 vortex shedding cycles after the flow had developed, which was 33000 time steps. Before applying our computational DNS approach to a cylinder covered by a porous layer, we have first tested it extensively for the well studied case of flow around a solid cylinder at subcritical Re. For this case, our results (e.g. time averaged velocity profiles and turbulence spectra) were in very good agreement with results published by Ma et al. [6] and Tremblay [7.

\subsection{Micro-scale DNS}

For the micro-scale simulations of the flow around the textile fibers we again used Fluent. We created a so-called virtual textile, schematically presented in the right-hand part of Fig. 1. Since NBC clothing consists of woven textile, the geometric structure is ordered with only minor irregularities. The shape, diameter and distribution of the textile fibers were based on average values observed in a microscopic image of an actual NBC protective textile. A 3-dimensional flow domain was simulated, with the virtual textile plane halfway and parallel to the inflow and outflow boundary of the domain. The inflow boundary conditions were obtained from the meso-scale DNS simulations, from which it was found that the flow has laminarized in the vicinity of the textile. Periodic boundary conditions were applied at the edges of the virtual textile. The total size of the computational domain was about $200 \mathrm{k}$ cells. Due to the low fiber diameter and low velocity $\left(10^{-2} \leq \operatorname{Re} \leq 10^{1}\right.$, with $\operatorname{Re}$ based on the fiber diameter), the flow remains laminar and steady. A comprehensive study of the influence of different fiber volume fractions and different inflow conditions on the pressure drop and permeability has been performed. As outcome of these studies we obtained realistic values for the range of the textile resistances and the range of the superficial velocities through the porous layer. These values have subsequently been used as input for the meso-scale DNS and meso-scale RANS simulations. 


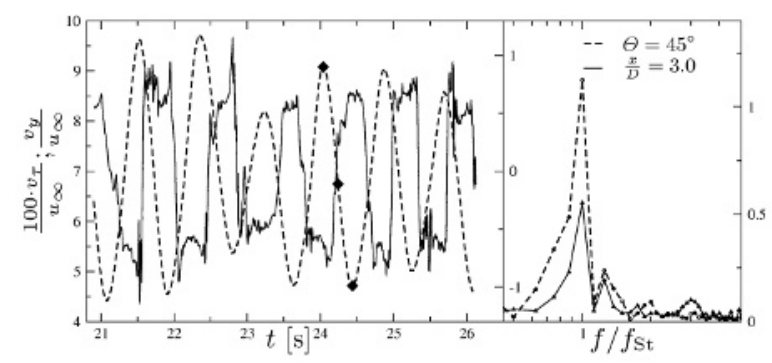

Fig. 2. Dimensionless velocity $v_{y}$ in the wake at $x / D=3$ and tangential velocity $v_{\tau}$ inside the air gap at $\Theta=45^{\circ}$, both in time (left) and Fourier (right) space

\subsection{Meso-scale RANS}

Two-dimensional engineering simulations of flow, heat and mass transfer at meso-scale were performed earlier in a comprehensive parameter study, details of which have been reported in [1. Based on this parameter study, a correlation has been formulated, predicting heat and mass flux through the protective clothing as a function of clothing properties and flow conditions. Here we compare these earlier results to results from present DNS.

\section{Results and Discussion}

\subsection{Meso-scale DNS}

As expected, the flow inside the air gap (i.e. in the space between the outer porous cylinder and the inner solid cylinder) is transient. Its dynamics is due to the vortex shedding in the wake of the outer flow. In Fig. 2 2 the velocity inside the air gap is compared to the velocity in the wake. The inner flow velocity is orders of magnitude smaller than the outer flow, but exhibits the same periodic behavior, as can be seen from the figure's left-hand part. It can also be seen that only the low shedding frequency is pronouncedly present in the inner flow, whereas higher frequencies are filtered out. The right-hand part of the graph confirms that the frequency of the flow inside the air gap is locked to the frequency of vortex shedding in the outer flow.

Fig. 3 (left) shows the tangential velocity component along the air gap centerline. The velocity is presented as time averaged quantity, together with three instantaneous time realizations. The latter were taken at instances corresponding to the maximal, intermediate and minimal value of the velocity at $\Theta=45^{\circ}$, respectively. The inset figure is a simplified representation of the periodic inner flow behavior from Fig. 2, with the three time realizations indicated by black dots. The velocity distribution in the air gap at different time instances confirms its periodic nature. The time averaged velocity profile is in good agreement with our experimental data obtained by Laser Doppler Anemometry [1]. 

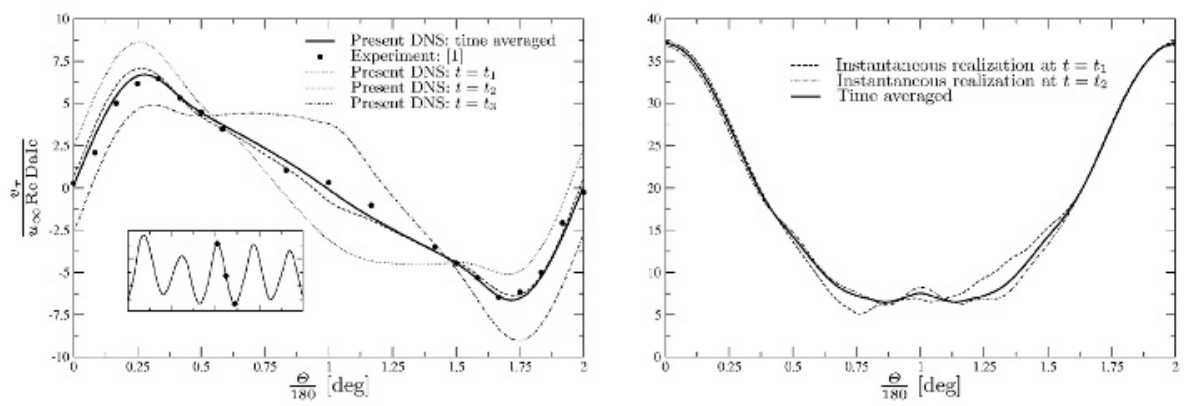

Fig. 3. Dimensionless, tangential velocity component at the air gap centerline (left) and local $\mathrm{Nu}$ number (right) both as a function of angle

Fig. 3(right-hand part) presents the Nusselt number, which is a dimensionless measure of heat transfer from the solid cylinder surface to the air, as a function of angle. The time averaged quantity is compared to the instantaneous ones gathered at two different time instances. The Nusselt number distribution shows that the highest heat flux to the solid cylinder occurs in the vicinity of the front stagnation point, as expected. The heat transfer is strongly correlated to the radial velocity component, with high heat transfer at locations were there is a high inward air velocity.

\subsection{Micro-scale DNS}

For the virtual textile, simulations for varied $\operatorname{Re}=\frac{\varrho\langle v\rangle 2 r_{\mathrm{f}}}{\mu}$ were conducted, where $r_{\mathrm{f}}$ is fiber radius. Note that here $\langle v\rangle$ is the velocity in the vicinity of the textile, which is orders of magnitude smaller than the free stream velocity. Furthermore, at fixed Re, simulations were conducted for a range of textile porosities $\epsilon$, defined as the ratio of the open frontal area to the total frontal area of the textile. When the textile is considered to be a 2-D square array of cylinders of radius $r_{\mathrm{f}}$, at a mutual half distance $\delta$, then $\epsilon$ is given by

$$
\epsilon=\frac{\delta^{2}}{\left(r_{\mathrm{f}}+\delta\right)^{2}} \quad \text { or } \quad \frac{\delta}{r_{\mathrm{f}}}=\frac{\sqrt{\epsilon}}{1-\sqrt{\epsilon}}
$$

In the left-hand part of Fig. 4, the pressure gradient $\nabla P$ through one specific textile as a function of Re is presented. Numerical results are compared to the unpublished experimental data obtained at the Prins Maurits Laboratory of the Netherlands Organization for Applied Scientific Research (TNO). It is clearly seen that for two decades of Re the pressure gradient through the textile depends linearly on Re, in agreement with Darcy's law, which assumes that the pressure drop is due to viscous effects only. Inertia effects become important for larger values of Re, and a super-linear dependence of the pressure gradient on Re is observed. 

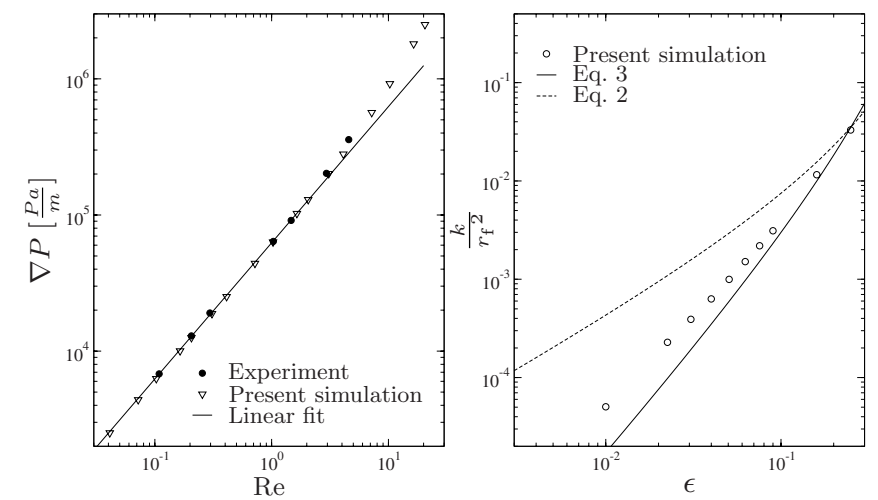

Fig. 4. Computed pressure gradient versus Re (left) and dimensionless permeability as a function of textile porosity (right)

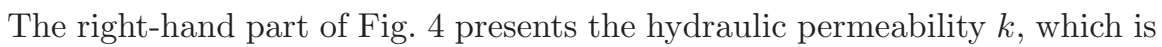
a measure of the fluid conductance in a porous layer, as a function of porosity. It is related to the pressure gradient by Darcy's law $\nabla P=\mu \frac{\langle v\rangle}{k}$. Based on a simple analysis combining Stokes flow and mass continuity, Clague et al. [8] proposed the following scaling estimate for the hydraulic permeability of an infinite square array of cylinders:

$$
k=\text { const } \cdot \delta^{2} \quad \text { or } \quad \frac{k}{r_{\mathrm{f}}^{2}}=\text { const } \cdot \frac{\epsilon}{(1-\sqrt{\epsilon})^{2}}
$$

This result was obtained using $\langle v\rangle$ as the characteristic scale for the velocity through the array. This is reasonable for large porosities, but for low porosities it is more appropriate to use $\frac{\langle v\rangle}{\epsilon}$ as velocity scale. This leads to:

$$
k=\text { const } \cdot \delta^{2} \epsilon \quad \text { or } \quad \frac{k}{r_{\mathrm{f}}{ }^{2}}=\text { const } \cdot \frac{\epsilon^{2}}{(1-\sqrt{\epsilon})^{2}}
$$

By fitting the constant in Eq. 3 we found good agreement to our numerical data, as can be seen in the right-hand side of Fig. 4 The small deviation for $\epsilon \rightarrow 0$ is to be expected, since friction is becoming important here. It is clear that Eq. 3 which resembles to the Kozeny-Carmen equation for flow through a packed bed of particles, represents a better scaling estimate than Eq. 2.

\subsection{Meso-scale RANS}

Ultimately, we wish to develop an engineering model of NBC protective clothing at the full body macro scale [9], based on lumped parameter descriptions of the textile and on RANS modeling of the air flow. Earlier [1] we performed such simulations at the meso-scale (see Fig. 1), focusing on the flow in the air gap 

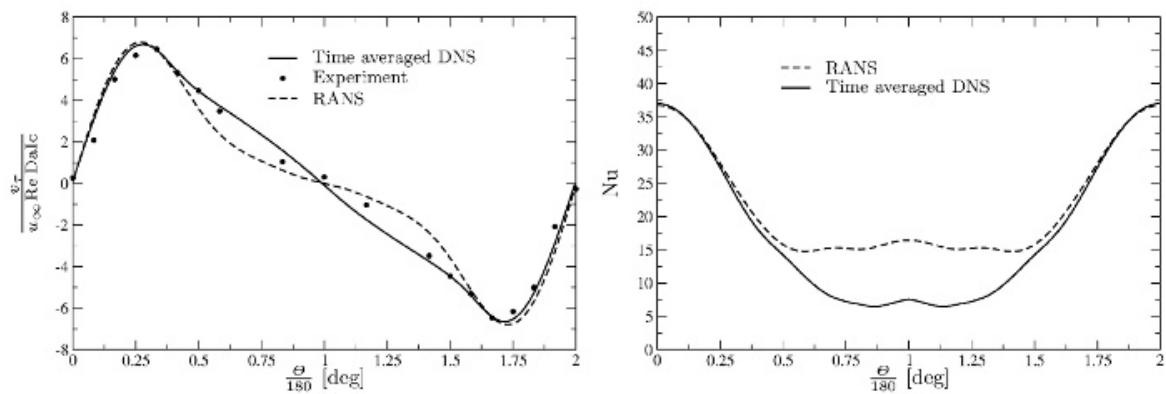

Fig. 5. Tangential velocity component at the air gap centerline (left) and local $\mathrm{Nu}$ distribution (right)

and on heat and mass transfer from the flowing air to the inner cylinder surface. In the present paper we validate these results against novel meso-scale DNS data. As shown in [1, for the studied cases there is a one-to-one correspondence between the Nusselt number for heat transfer and the Sherwood number Sh for mass transfer. Because of this similarity, only results for heat transfer will be presented here.

In the left hand side of Fig. 5 the dimensionless tangential velocity component in the air gap is presented as a function of angle. The velocity distribution obtained from RANS is compared to velocity profile obtained from DNS after time averaging (shown earlier in Fig. 3) and to our experimental LDA data. The overall performance of the RANS model is satisfactory. The maximum velocity is well predicted, a slight under prediction occurs for $90^{\circ}<\Theta<270^{\circ}$.

The right-hand side of Fig. 5 presents a comparison between time averaged $\mathrm{Nu}$ numbers obtained from DNS and RANS computations. In the vicinity of the front stagnation point $\left(-90^{\circ}<\Theta<90^{\circ}\right)$, where heat transfer is largest and most critical, the agreement is very good. In the downstream region, $90^{\circ}<\Theta<270^{\circ}$, where the flow in the gap is unsteady due to the vortex shedding in the outer flow, the steady-state simplification of RANS leads to significant over-predictions of the heat transfer. Nevertheless, averaging the $\mathrm{Nu}$ number over all angles, gives a satisfactory agreement between DNS $(\langle\mathrm{Nu}\rangle=18)$ and $\operatorname{RANS}(\langle\mathrm{Nu}\rangle=20)$ results.

From the above comparisons, we conclude that the RANS model performs sufficiently accurate for engineering purposes, particularly when we focus on global (non-local) analyses of heat and mass transfer. We used this model to study the influence of the free-stream air velocity, the hydraulic permeability and thickness of the porous layer, and the distance between the inner and outer cylinder on the average $\mathrm{Nu}$ number at the inner cylinder surface. Simulations were carried out for typical values of the porous layer permeability, expressed in dimensionless form as DaIc $=\frac{k}{4 R_{\mathrm{p}} \cdot r_{\mathrm{f}}}$, and the dimensionless air gap thickness Ig. Based on the results from 2-D RANS simulations, an empirical correlation, that can be used as an engineering tool, was proposed and reported in 11. 


\section{Conclusions}

The large differences of scale between the phenomena of interest necessitate a multi-scale approach for modeling the performance of NBC protective clothing. In our attempts to ultimately develop a computational model for the design and evaluation of NBC protective clothing at the full body macro scale, we performed various studies of flow, heat and mass transfer at micro and meso scales. Direct Numerical Simulations at meso-scale of the turbulent flow around a sheathed cylinder (mimicking a clothed limb) showed that, for the free flow velocities of interest, the flow underneath the clothing is laminar and periodic, with a frequency locked to that of the vortex shedding. For the range of clothing permeabilities of interest, the velocity of the flow through the clothing was found to be orders of magnitude smaller than the free stream velocity. Direct Numerical Simulations at micro-scale of the laminar flow around the textile fibers showed that, for the range of air velocities and textile porosities of interest, the pressure drop over the textile can be accurately described by Darcy's law. A simple scaling rule was proposed for the hydraulic permeability as a function of porosity and fiber thickness. The above findings from micro and meso scale DNS validate the simplifications that were made in a RANS type engineering model. From a comparison between meso scale DNS and RANS simulations, it is concluded that the RANS models has shortcomings in accurately predicting local Nusselt and Sherwood numbers, but performs satisfactorily accurate in predicting global heat and mass transfer. The model is now ready to be used for macro-scale full body studies.

\section{References}

1. Sobera, M., Kleijn, C., Brasser, P., van den Akker, H.: Convective heat and mass transfer to a cylinder sheathed by a porous layer. AIChE Journal 49 (2003)

2. Zdravkovich, M.: Flow around circular cylinders. Oxford University Press, (1997)

3. Norberg, C.: Efects of Reynolds number and low-intensity freestream turbulence on the fow around a circular cylinder. TR 87/2, Department of Applied Thermodynamics and Fluid Mechanics, Chalmer University of Technology, Sweden (1987)

4. Ong, L., Wallace, J.: The velocity field of the turbulent very near wake of a circular cylinder. Exp. Fluids 20 (1996) 441-453

5. Breuer, M.: Large eddy simulation of the subcritical flow past a circular cylinder: numerical and modeling aspects. Int. J. Numer. Meth. Fluids 28 (1998) 1281-1302

6. Ma, X., Karmanos, G.S., Karniadakis, G.: Dynamics and low-dimensionality of a turbulent near wake. J. Fluid Mech. 410 (2000) 29-65

7. Tremblay, F.: Direct and large-eddy simulation of flow around a circular cylinder at subcritical Reynolds numbers. PhD thesis, Munich University of Technology, Germany (2001)

8. Clague, D., Kandhai, B., Zhang, R., Sloot, P.: Hydraulic permeability of (un)bounded media using lattice boltzman method. Physical Review E 61 (2000) 616-625

9. Barry, J., Hill, R., Brasser, P., Sobera, M., Kleijn, C., Gibson, P.: Computational fluid dynamics modeling of fabric systems for intelligent garment design. MRS Bulletin 28 (2003) 568-573 